\title{
Two Desynaptic Mutants of Rhoeo spathacea (Commelinaceae)
}

\author{
Armando Garcia-Velazquez \\ Especialidad de Genetica Iregep, C.P. Montecillo, Edo. de, C.P.56230, Mexico
}

Accepted June 20, 2001

\begin{abstract}
Summary Two desynaptic plants causing dissociation of pairing chromosomes were found among plants collected in wild populations of Rhoeo spathacea. One of those plants is of the spathacea variety and was identified as GAVA YT-1, while the other was found to be of the concolor variety and was identified as GAVA C-2. Both plants were found to be diploids with $2 n=12$ and displayed 2 cytotypes: $9 m+3 s m$ and $4 m+8 s m$, respectively. GAVA C-2 is the first desynaptic plant observed of the concolor variety. The mutant plants formed univalents in all PMC at MI. GAVA YT-1 formed 12 univalents in $84.9 \%$ of PMC and $89.7 \%$ in PMC of GAVA C-2. Microspores from GAVA YT-1 had chromosome from $n=5$ to $n=12$, and microspores produced by GAVA C- 2 showed $n=8$ to $n=18$. The GAVA C-2 plant produced $21.3 \%$ unreduced microspores. There were large differences in pollen stainability between the 2 desynaptics: GAVA YT- 1 with $17.2 \%$ and GAVA C- 2 with $55.5 \%$.
\end{abstract}

Key words Desynaptic, Meiotic mutants Rhoeo.

Rhoeo spathacea (Swartz) Stern is the best known complex interchange heterozygote capable of forming a complete ring of all its 12 chromosomes during meiosis. Three morphotypes are recognized within the species; 1) var. spathacea, the typical form with bicolored leaves, dark green above and purple beneath, 2) var. concolor with completely green leaves (Baker and Mertens 1975, Verma and Ohri 1979), and 3) var. variegata, an uncomon form with leaves with longitudinal yellowish stripes on the otherwise dark green upper side (Lin 1980). In our living collection, plants of this variety has been developed as chimeras on var. spathacea (Garcia unpublished data). Several cytologists (Darlington 1929, Sax 1931, Lin and Paddock 1973, Mertens 1973, Baker and Mertens 1975) have noted the unique chromosome cytology of Rhoeo. They have found a diploid with 12 chromosomes, each chromosome involved in reciprocal translocations with 2 other chromosomes. In higher plants the interchange heterozygotes must be identified cytologically by the association of chromosomes involved or genetically by linkage of genes that are normally independent (Burnham 1956). Desynaptic Rhoeo mutants have been observed recently. The observed mutant meiosis is a complete breakdown of conventional meiotic behavior of Rhoeo (Garcia 1991, 1994, 1995). Selfed progeny of one of such desynaptics (and SDR) included 123 seedlings. Of these, 90 diploids and one acrotrisomic (Garcia 1995) unexpectedly reverted desynapsis to ring-forming, and 32 hyperploids ( $2 n=24$ and $2 n=25$ ) behave as desynaptics at MI (Garcia 2000). Preliminary data for another selfed progeny of a desynaptic mutant, currently being collected, differ from expected homozygous desynaptic mutant. Synaptic genes have been reported in a number of crop plants as recessive (Koduro and Rao 1981, Kaul and Murthy 1985) and most all in diploid plants (Singh 1993). Additional meiotic mutants will be useful in understanding desynaptic inheritance in Rhoeo.

The purpose of this report is to extend the description of meiotic behavior with another 2 desynaptic mutants collected from wild populations in order to further understand the meiotic behavior of Rhoeo. 


\section{Materials and methods}

In 1992, Rhoeo spathacea var. spathacea and var. concolor, were collected in Temozon and the Ex-Hacienda Xcumpich, Merida, Yucatan (Mexico). Plants were identified as GAVA YT-1 and GAVA C-2, respectively. Plants collected from wild populations are now growing as live collections at Colegio de Postgraduados.

\section{Cytological methods}

Chromosome observations were performed on chromosome preparations made on root tips, following a procedure of Garcia (1995). Ten cells of high quality were drawn with a Zeiss tube attachment with $3400 \times$ magnification on a FOMI III microscope. Metaphase chromosomes were identified simultaneously using relative length (L\%) and arm ratio (r) as proposed by Tjio and Hagberg (1951). For meiotic analysis of pollen mother cells (PMC), inflorescences were collected when central flowers opened. Anthers of suitable size were squashed directly in $c a .1 .8 \%$ propionic orcein and heated gently, then carefully squashed again to spread out the chromosomes. Metaphase I and anaphase I were scored for chromosome behavior. Young microspores were used for chromosome counts at first mitotic division. Pollen grains stained with propionic orcein were counted.

Photomicrographs were taken on Kodalith film using a Zeiss FOMI III microscope and planopochromatica plane $100 \times$ oil immersion objective.

\section{Results and discussion}

In both of the desynaptic plants the chromosome number in somatic cells observed was $2 n=12$. This agrees with previous chromosome counts in Rhoeo (Garcia 1991, 1994). In the karyotype of each of the plants there were no 2 wholly homologous chromosomes, as has been observed in Rhoeo (Natarayan and Natarayan 1972, Garcia 1991, 1994).

\section{Karyotype}

The 12 chromosomes were numbered and arranged in decreasing order of relative length (L\%) (Fig. 1, Table 1). In this table arm ratio (r) is also included. It can be seen that there are bimodal karyotypes including metacentric and submetacentric chromosome (Table 1).

The karyotype of plant GAVA YT-1 consisted of 9 metacentric chromosomes (numbers 1, 2, 3, $4,5,6,7,10,12$ ), and 3 submetacentric chromosomes (numbers $8,9,11$ ). The karyotype of plant GAVA C-2 contained 4 metacentric chromosomes (numbers 1, 2, 3, 4) and 8 submetacentric chromosomes (numbers 5, 6, 7, 8, 9, 10, 11, 12), following the nomenclature of Levan et al. (1964). In the karyotype of GAVA C-2 chromosome 8 bears a secondary constriction in the short arm giving rise to a small spherical satellite.

The bimodal karyotype coincides with previous reports, and it can therefore be said that there is no standard karyotype of Rhoeo. In fact, there is a great deal of variability in Rhoeo karyotypes. Natarajan and Natarajan (1972) observed a karyotype consisting of 6 metacentric and 6 submetacentric chromosomes. Garcia $(1991,1994)$ observed karyotypes with 8 metacentric and 4 submetacentric chromosomes, respectively. Garcia (1995) postulated that unequal interchanges occurring in terminal regions of Rhoeo chromosomes result in karyotype variability. An extreme case was the presence of an acrocentric chromosome in a trisomic one $(2 n=13)$ (Garcia 1995). Selected metaphase chromosome plates with good spreading result in accurate karyotype analysis. The simultaneous use of 2 variables (Fig. 2, Table 1) allows a better comparison of chromosome complements in both desynaptic plants. Minor differences in relative length (L\%) and arm ratio (r) from one cell to another were rather common. The use of the method proposed by Tjio and Hagberg (1951) was very useful and accurate in karyotyping Rhoeo. These results confirm that in Rhoeo 
karyotypes there are no 2 homologous chromosomes.

\section{Meiosis}

The early prophase of meiosis of Rhoeo is not suitable for meiotic analysis. However, up to diplotene, chromosome association capable of forming a complete ring or chain of all 12 chromosomes can be seen. In both GAVA YT-1 and GAVA C-2 plants, univalents were caused by desynapsis of the initially paired chromosomes. At metaphase I in such desynaptics there is no metaphase plate. Instead, chromosomes are scattered randomly throughout the cytoplasm (Fig. 2).

GAVA YT-1 had at least 8 univalents and up to 12 univalents in $85 \%$ of 578 PMC (Table 1). GAVA C-2 exhibited a higher percentage $(90 \%)$ of PMC with 12 univalents (Table 1). This was the first report of a concolor var. plant presenting desynapsis. The concolor var. exhibits ring-forming, bivalent forming (Wimber 1968) and desynaptic meiotic behavior. In GAVA YT-1 and GAVA C-2 plants no rings or chains were observed in any cell. Garcia (1991) described the first desynaptic mutant in Rhoeo in GAVA 1.1, which exhibited up to 12 univalents in nearly $24 \%$ of about 3000 PMC analyzed. Synaptic mutants exhibited abnormal meiosis and reduced fertility, the degree and expression of gene action varied. Another desynaptic mutant, GAVA 3, showed 12 univalents in $55.6 \%$ of the analyzed cells (Garcia 1995). In blueberry ( $V$. darrowi) 2 genotypes

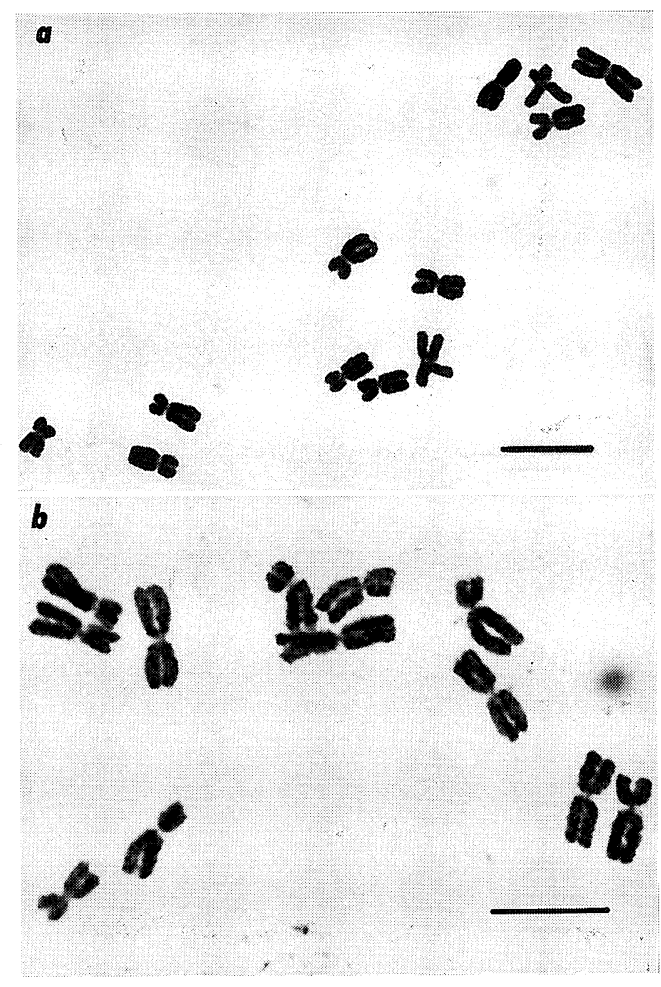

Fig. 1. Somatic chromosomes of desynaptic Rhoeos. a. GAVA YT-1, b. GAVA C-2. Scale $=10 \mu \mathrm{m}$.

Table 1. Relative length (L\%) and arm ratio (r) of chromosomes in GAVA YT-1 and GAVA C-2 desynaptic Rhoeo spathacea

\begin{tabular}{|c|c|c|c|c|}
\hline \multirow{2}{*}{$\begin{array}{l}\text { Chromosome } \\
\text { number }\end{array}$} & \multicolumn{2}{|c|}{ Relative length (L\%) } & \multicolumn{2}{|c|}{ Arm ratio (r) } \\
\hline & GAVA YT-1 & GAVA C-2 & GAVA YT-1 & GAVA C-2 \\
\hline 1 & $10.16 \pm 1.16$ & $10.73 \pm 0.75$ & $1.27 \pm 0.43 \mathrm{~m}$ & $1.20 \pm 0.10 \mathrm{~m}$ \\
\hline 2 & $9.60 \pm 1.21$ & $9.88 \pm 0.28$ & $1.12 \pm 0.40 \mathrm{~m}$ & $1.41 \pm 0.29 \mathrm{~m}$ \\
\hline 3 & $9.27 \pm 0.92$ & $9.46 \pm 0.24$ & $1.46 \pm 0.04 \mathrm{~m}$ & $1.23 \pm 0.11 \mathrm{~m}$ \\
\hline 4 & $8.82 \pm 0.84$ & $9.16 \pm 0.26$ & $1.34 \pm 0.49 \mathrm{~m}$ & $1.27 \pm 0.33 \mathrm{~m}$ \\
\hline 5 & $8.67 \pm 0.74$ & $8.81 \pm 0.25$ & $1.48 \pm 0.36 \mathrm{~m}$ & $1.72 \pm 0.61 \mathrm{sm}$ \\
\hline 6 & $8.44 \pm 0.72$ & $8.66 \pm 0.30$ & $1.47 \pm 0.48 \mathrm{~m}$ & $1.85 \pm 0.84 \mathrm{sm}$ \\
\hline 7 & $8.08 \pm 0.76$ & $8.26 \pm 0.26$ & $1.41 \pm 0.52 \mathrm{~m}$ & $1.72 \pm 0.47 \mathrm{sm}$ \\
\hline 8 & $8.04 \pm 0.77$ & $7.81 \pm 0.19$ & $1.72 \pm 0.45 \mathrm{sm}$ & $1.90 \pm 0.23 \mathrm{sm}$ \\
\hline 9 & $7.82 \pm 0.88$ & $7.63 \pm 0.19$ & $1.65 \pm 0.46 \mathrm{sm}$ & $1.76 \pm 0.31 \mathrm{sm}$ \\
\hline 10 & $7.40 \pm 0.77$ & $7.08 \pm 0.45$ & $1.48 \pm 0.58 \mathrm{~m}$ & $1.86 \pm 0.63 \mathrm{sm}$ \\
\hline 11 & $7.07 \pm 0.77$ & $6.72 \pm 0.42$ & $1.92 \pm 0.39 \mathrm{sm}$ & $1.86 \pm 0.34 \mathrm{sm}$ \\
\hline 12 & $6.62 \pm 0.68$ & $5.93 \pm 0.56$ & $1.56 \pm 0.96 \mathrm{~m}$ & $1.91 \pm 0.85 \mathrm{sm}$ \\
\hline
\end{tabular}




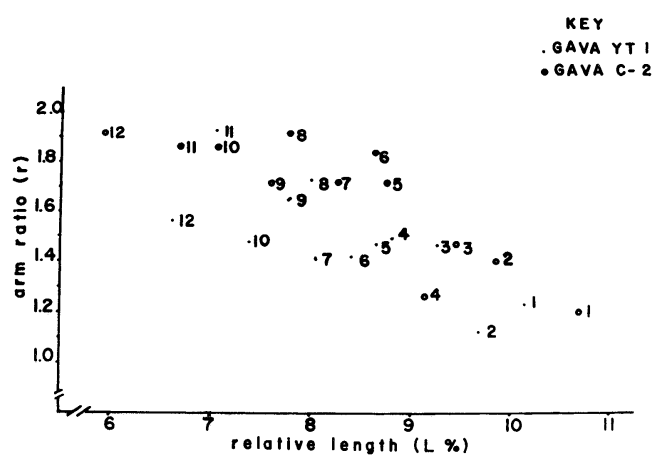

Fig. 2. Graph of relative length ( $\mathrm{L} \%)$ and arm ratio (r) of chromosome measurements in Table 2.

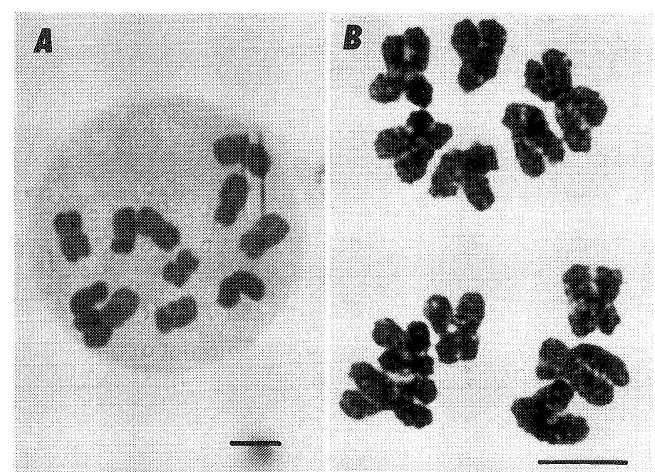

Fig. 3. Meiotic behavior in desynaptic PMC. A) 12 univalents at M I. B) Distribution 6:6 at A I. Scale $=5 \mu \mathrm{m}$.

showed differences in the expression of desynapsis: complete desynapsis (Qu and Vorsa 1994) and rare (Vorsa and Ortiz 1992). A desynaptic mutant in maize $\left(\mathrm{dsy}^{1}\right)$ has a high frequency of univalents and rare bivalents of diplotene-diakinesis was nearly sterile owing apparently to disjunctive irregularities (Maguire et al. 1993). Miller (1963) observed mutations of maize at pachytene to metaphase I and found zero to total synapsis of homologous chromosomes. McCoy (1982) indicated that many meiotic mutants have incomplete expressivity. This could be the reason that desynaptic mutants have different degrees of expressivity of desynapsis. Therefore, it could be said that desynaptic Rhoeo plants exhibit different degrees of penetration or expressivity of desynapsis. Other factors that may affect meiotic behavior could be the differential action of meiotic pairing control genes. In the present study, GAVA YT-1 showed 85\% PMC with $12_{1}$, while GAVA C-2 had 90\% PMC with $12_{1}$. In hybrid crosses of hexaploid ecotypes of tall fescue (Festuca arundinacea, $2 n=6 x=42$ ), desynaptic meiosis with concomitant abnormalities resulted in laggrads with divided and misdivided univalents (Jauhar 1993). A requirement of successful meiosis, in addition to chiasma maintenance until anaphase $\mathrm{I}$, is the maintenance of adhesion of sister centromeres (Maguirre et al. 1993). However, it is observed in the present study that, even when chiasma maintenance is not observed until anaphase I, the strong adhesion of sister centromeres is observed until anaphase II and ensures a high percentage of haploid chromosome number in the produced microspores.

\section{Anaphase I}

There is no concordance between the high percentage of univalents due to chiasmata failure and the regular disjunction at A I. For example, in GAVA YT-1 nearly $85 \%$ of the PMC examined had 12 univalents, but about $56 \%$ of anaphase I cells had 6:6 disjunction. for GAVA C-2 nearly $90 \%$ of metaphase I cells had 12 univalents with $40 \%$ of anaphase cells having $6: 6$ disjunction. Therefore, it seems that there is a force that aligns chromosomes. In flea beetle (Alagoasa bicolor) sex univalents $(\mathrm{X}, \mathrm{Y})$ are independent of each other and of autosomes and oriented synthetically, and segregate to opposite poles probably because of spindle elongation (Kupfer and Wise 2000).

Lagging chromosomes $(5: 1: 6)$ were more numerous in the GAVA C-2 plant with $15.58 \%$, while GAVA YT-1 showed 10.33\% lagging distribution (Table 1). The GAVA C-2 plant also exhibited the higher percentage of other distributions or more irregularities, $21.52 \%$ of the PMC had such chromosome distribution (Table 1). GAVA YT-1 had about half as many other chromosomal distributions as plant GAVA C-2. According to Lin and Paddock (1973), the usual mode of chromosome distribution of Rhoeo is that adjacent chromosomes pass to opposite poles and alternate ones to the same pole. In ring-forming, generally Rhoeo chromosomes are distributed equally (6:6), at A I in varying percentages: 70\% (Walters and Gerstel 1948), 71.6\% (Lin and Paddock 1973), 59.5\% 
Table 2. Chromosome associations at M I and chromosome segregation at A I in desynaptics of Rhoeo spathacea

\begin{tabular}{|c|c|c|c|c|c|}
\hline \multirow{3}{*}{$\begin{array}{l}\text { Meiotic } \\
\text { stage }\end{array}$} & \multirow{3}{*}{ Univalent/Cell } & \multicolumn{4}{|c|}{ Observed PMC } \\
\hline & & \multicolumn{2}{|c|}{ GAVA YT-1 } & \multicolumn{2}{|c|}{ GAVA C-2 } \\
\hline & & Number & $\%$ & Number & $\%$ \\
\hline \multirow[t]{6}{*}{ M I } & 12 & 491 & 84.95 & 703 & 89.78 \\
\hline & 10 & 36 & 6.23 & - & - \\
\hline & 9 & 18 & 3.11 & - & - \\
\hline & 8 & 33 & 5.70 & - & - \\
\hline & others & - & - & 80 & 10.22 \\
\hline & total & 578 & - & 783 & - \\
\hline \multirow[t]{5}{*}{ A I } & $6: 6$ & 120 & 56.34 & 142 & 40.20 \\
\hline & $5: 1: 6$ & 22 & 10.32 & 55 & 15.58 \\
\hline & $5: 7$ & 49 & 23.00 & 80 & 22.66 \\
\hline & others & 22 & 10.32 & 76 & 21.52 \\
\hline & & 213 & & 353 & \\
\hline
\end{tabular}

(Mertens 1973), and 58.7\% (Lin 1980). Garcia (1999) proposed that Rhoeo constitutes a dikaryon that includes subgenomes A and B, each of which is present in both poles at $\mathrm{T}$ I. These results support the idea that chiasmata are not necessary for chromosome distribution as univalents, which are usually present in large numbers and move to each pole, and the $6: 6$ disjunction (56\% in GAVA YT-1 and 40\% in GAVA C-2, Table 1) deviated significantly from being random.

\section{Pollen cytology}

By using pollen stainability in propionic orcein $1.8 \%$ as the index of fertility, high variability was observed among plants. GAVA C-2 plant had 770 stainable (56\%) and normal-shaped pollen grains, out of 1387. GAVA YT-1 had very low pollen stainability, as only 1551 out of 8987 $(17.26 \%)$ stained and were well-filled pollen grains. These results in the pollen fertility index are probably due to the high genetic variability in Rhoeo spathacea plants and not to the balance in chromosome number.

Chromosome counts at the microspore stage also exhibited great variability, the GAVA C-2 plant showed a chromosome number ranging from $n=5$ to $n=18$ (Table 2) with $n=6$ in almost $65 \%$ of 165 microspores observed. Microspores of GAVA YT-1 showed a chromosome number from $n=5$ to $2 n=12$, in this case, $70 \%$ of the microspores had $n=6$. Therefore, both GAVA C- 2 and GAVA YT-1 desynaptic plants produced almost haploid microspores (Table 2). But GAVA C-2 produced $21.34 \%$ unreduced microspores. Based on these results, it can be said that the mechanisms governing $2 n$ pollen production is a second division restitution (SDR) via failure of cytokinesis. These results coincide with previous reports on Rhoeo (Garcia 1991, 1995). In desynaptic blueberry (genus Vaccinum), fertile $2 n$ gametes are produced when univalents undergo homologous centromere disjunction at A I. No second division occurs (Vorsa and Ortiz 1992). Therefore, in blueberry unreduced gametes are produced via FDR, while in Rhoeo $2 n$ gametes are produced by SDR.

Even when there are a variety of methods used to detect $2 n$ gametes, the easiest and most exact method is the direct examination of chromosome number. The presence of giant grains has been frequently used as an indication of the production of $2 n$ pollen (Bretagnolle and Thompson 1995). However, in this study giant pollen grains with $n=7$ and $2 n=12$ and "jumbo" (possibly $4 n$ ), and also small ones with $n=7$, were observed. Fortunately, in Rhoeo it is possible to count chromosomes in pollen grains (Garcia 1991, 1995, 1999).

In previous studies of desynapsis in Rhoeo (Garcia 1991, 1995) it has been observed that $2 n$ pollen results from the fusion of nuclei with 6 chromosomes at second division. Those nuclei were fused due to aberrant cytokinesis resulting from a failure in the equational cell wall. However, it is 
possible that $2 n=18$ may result from failure in both reductional and equational cell walls. Another possibility for production of $4 n$ gametes is FDR, but more observations are required. The high frequency of $2 n$ pollen produced by desynaptics, GAVA 1.1. (47.8\%, Garcia 1991), GAVA 3 (34\%, Garcia 1994), and GAVA C-2 (21\% this report) suggests genetic control of this mechanism. Apparently, such a mechanism is not present in GAVA YT-1, which produced less than $2 \% 2 n$ gametes. Two different genotypes of blueberry (Vaccinium darrowi), one "desynaptic" (Qu and Vorsa 1999) and an "aneuploid" (Vorsa and Ortiz 1992) produce $2 n$ gametes "by a mechanically similar process".

\section{References}

Baker, R. F. and Mertens, T. R. 1975. Meiosis in variegated and anthocyninless varieties of Rhoeo. J. Heredity 66: $381-383$. Bretagnolle, F. and Thompson, J. D. 1995. Gametes with the somatic chromosome number. Mechanisms of their formation and role in the evolution of autopolyploid. New Phytol. 129: 1-22.

Burnham, C. R. 1956. Chromosomal interchanges in plants. Bot. Rev. 22: 419-552.

Darlington, C. D. 1929. Chromosome behavior and structural hybridity in Tradescantia. J. Genet. 21: 207-286.

Garcia, V. A. 1991. Cytogenetical studies in Rhoeo spathacea (Comelinaceae) I. A Desynaptic and second division restitution mutant. Genome 34: 895-899.

- 1994. A desynaptic mutant in Rhoeo spathacea (Commelinaceae). Cytologia 59: 399-404.

- 1995. Cytogenetical studies in Rhoeo spathacea (Commelinaceae) II. Characterization of an acrotrisomic plant. Cytologia 60: 319-327.

- 1999. Cytogenetical studies in Rhoeo spathacea (Commelinaceae) III. Mixoploid derived from a desynaptic plant. Cytologia 64: 45-49.

- 2000. Cytogenetical studies in Rhoeo spathacea (Commelinaceae) IV. Ring-forming diploid and desynaptic tetraploid sibs. Cytologia 65: 179-197.

Jauhar, P. P. 1993. Cytogenetics of the Festuca-Lolium Complex. Springer Verlag. pp. 57-69.

Kaul, M. L. and Murthy, T. G. K. 1985. Mutant genes affecting higher plant meiosis. Theor. Appl. Genet. 70: $449-466$.

Koduro, P. R. K. and Rao, M. K. 1981. Cytogenetics of synaptic mutants in higher plants. Theor. Appl. Genet. 59: 197-214.

Kupfer, H. and Wise, D. 2000. Behavior of sex chromosomes, autosomes, and the spindle during nonrandom segregation in a flea beetle. Genome 43: 521-527.

Levan, A., Fredga, K. and Sandberg, A. A. 1964. Nomenclature for centromeric position on chromosomes. Hereditas 52: 201-220.

Lin, Y. J. 1980. Complex heterocygosity in Rhoeo spathacea var. variegata. Genetics 94 (Abst.).

- and Paddock, E. F. 1973. Ring-position and frequency of chiasma failure in Rhoeo spathacea. Am. J. Bot. 60: 1023-1027.

Maguire, M. P., Riess, R. W. and Paredes, A. M. 1993. Evidence from a maize desynaptic mutant points to a probable role of synaptonemal complex central region components in provision for subsequent chiasma maintenance. Genome 36: $797-806$.

McCoy, T. A. 1982. The inheritance of $2 n$ pollen formation in diploid alfalfa, Medicago sativa. Can. Genet. Cytol. 24: 315-323.

Mertens, T. R. 1973. Meiotic chromosomes behavior of Rhoeo spathacea. J. Hered. 64: 365-368.

Miller, O. L. Jr. 1963. Cytogenetical studies in asynaptic maize. Genetics 48: 1445-1466.

Natarajan, A. T. and Natarajan, S. 1972. The heterochromatin of Rhoeo discolor. Hereditas 72: 323-330.

$\mathrm{Qu}, \mathrm{L}$. and Vorsa, N. 1999. Desynapsis and spindle abnormalities leading to $2 n$ pollen formation in Vaccinium darrowi. Genome 42: 35-40.

Sax, K. 1931. Chromosome ring formation in Rhoeo discolor. Cytologia 3: 36-53.

Sharma, R. K. and Reinbergs, E. 1974. Cytological analysis of desynaptic mutants in Barley (Hordeum vulgare L.). Cytologia 39: 77-81.

Singh, R. J. 1993. Plant Cytogenetics. C. R. C. Press, Boca Raton, Florida. pp. 391.

Tjio, L. H. and Hagberg, A. 1951. Cytological studies on some X-rays mutants of berley. An Estac. Exp. Aula Dei 2: 149-167.

Verma, S. C. and Ohri, D. 1979. Breakdown of the classical meiotic system in Rhoeo spathacea (Commelinaceae). Cytologia 44: $91-102$.

Vorsa, N. and Ortiz, R. 1992. Cytology of $2 n$ pollen formation in a blueberry aneuploid $(2 n=4 x+9=57)$. J. Hered. 83: 346-349.

Walters, M. S. and Gerstel, D. V. 1948. A cytological investigation of a tetraploid Rhoeo discolor. Am. J. Bot. 35: 141-150.

Wimber, D. E. 1968. The nuclear cytology of bivalent and ring-forming Rhoeos and their hybrids. Am. J. Bot. 55: $572-574$. 